\title{
Tetanus; a forgotten infection disease: a report of two cases
}

\author{
Ülkem Koçoğlu Barlas ${ }^{1 \oplus}$, Hasan Serdar Kıhtır ${ }^{1 \oplus}$, Osman Yeşilbaş ${ }^{1 \oplus}$, \\ Mey Talip Petmezci ${ }^{1 \oplus}$, Nihal Akçay ${ }^{1 \oplus}$, Ercüment Petmezci ${ }^{1 \oplus}$, Nevin Hatipoğlu ${ }^{2 \oplus}$, \\ Esra Şevketoğlu ${ }^{1 \oplus}$ \\ ${ }^{1}$ Division of Pediatric Intensive Care Unit, ${ }^{2}$ Department of Pediatrics, University of Health Science, Bakırköy Dr. Sadi Konuk Health \\ Training and Research Center, İstanbul, Turkey.
}

\begin{abstract}
Background. Tetanus is an infectious disease that can be seen in all age groups in underdeveloped and developing countries, where vaccination programs are inadequate. In developed countries, it is reported more frequently in the adult age group, where the protection of vaccination is diminished and the doses are delayed.

Case. In this report, we present generalized tetanus, which was observed in two male patients aged 12 and 6 years, admitted at different times, together with clinical course and treatment approaches. Both patients belong to different nationalities, who immigrated a couple of months before their application to our hospital. They applied with similar histories and complaints and were not vaccinated during infancy.

Conclusion. With the development of vaccination programs, this disease with high morbidity and mortality can be prevented.
\end{abstract}

Key words: clostridium tetani, tetanus, trismus.

Tetanus is an acute, often fatal disease that is caused by an exotoxin (tetanospasmin) produced by the anaerobic, gram-positive spore-forming bacterium Clostridium tetani. ${ }^{1}$ It can enter the body through any type of wound, such as a scratch or deep cut. ${ }^{2}$ Tetanospasmin is attached to the neuromuscular junction and migrates to motor neurons in the central nervous system (CNS) by retrograde axonal transport and disrupts the coordination of muscle movements by inhibiting the release of neurotransmitters. ${ }^{2}$ The microorganism is difficult to isolate and does not cause any obvious laboratory abnormality. Diagnosis is usually based on the trauma and tetanus immunization history, typical clinical findings and the exclusion of diseases that cause similar conditions. ${ }^{3}$ As a result of successful vaccination

\section{Ülkem Koçoğlu Barlas}

ulkemkocoglu@yahoo.com

Received 20th July 2017, revised 19th August 2017, 19th September 2017, 19th November 2017,

29th December 2018, accepted 6th April 2019. programs, the incidence of tetanus has been reduced to 0.02 per 100,000 population in Turkey. ${ }^{3}$ We present these two cases (three years apart) to draw attention to this disease, since it's diagnosis and treatment approaches are not well known by a new generation of physicians and it is more likely to be seen in our country with the increase in the immigrant population.

\section{Case 1}

A 12-year-old refugee male patient, who had a history of a punture on his right foot base after stepping on a nail ten days prior to admission, was admitted to the pediatric emergency department with complaints of difficulty in opening the jaw and back pain. On physical examination, in addition to lock-jaw, he had facial and abdominal muscle rigidity. He was hospitalized in the Pediatric Intensive Care Unit (PICU) with a preliminary diagnosis of tetanus. On admission to the intensive care unit, he was conscious, aware of his surroundings and agitated due to pain; he also had difficulty in 
speaking. The Glasgow Coma Scale (GCS) score was $15 / 15$. The respiratory and cardiovascular system examinations were normal. The patient's laboratory tests were normal. His medical history revealed that he had not been vaccinated against tetanus in childhood. 3000 International Units (IU) of tetanus immunoglobulin (TIG) was administered intramuscularly to the patient, who underwent tetanus vaccination in the emergency department, so that a half dose was applied to the area around the wound. His wound was superficial and clean, therefore no surgical wound debridement was required. Osteomiyelitis was also not considered because of normal imaging and negative infection markers as a result of orthopedics opinion. Diazepam was administered in combination with dexmedetomidine and fentanyl infusions for sedation and analgesia. Metronidazole, vancomycin and ceftazidime were started together because the bone and soft tissue infections could not be distinguished in theinjury area. Metronidazole and Ceftazidime were used for 10 days, Vancomycin was used for 14 days. On the second day of hospitalization, 3000 IU of TIG was readministered intramuscularly to the patient because diazepam did not reduce contractions. Then, intravenous magnesium sulphate and midazolam infusions were started. He was monitored in a silent and dark room. On the fifth day of hospitalization, he had difficulty in breathing because his body was stiff and therefore a total atelectasis developed in the left lung, which led him to be intubated. During his follow up treatment with deep sedation and analgesia it was decided to add muscle relaxant to his therapy. After intubation, analgesia was provided with fentanyl infusion whereas sedation was continued with midazolam and sodium thiopental infusions. Rocuronium infusion was started as a muscle relaxant. Baclofen was used enterally via the optimal dose-escalation strategy. On the tenth day of hospitalization, a sudden cardiac arrest developed in the patient while on mechanical ventilation and spontaneous circulation was achieved through approximately 10 minutes of cardiopulmonary resuscitation.
After rocuronium and sedative agents were discontinued weekly, he was awakened. However, treatment was maintained because severe contractions were observed again. At the end of the first month, the patient who still required mechanical ventilation underwent tracheostomy due to prolonged intubation. In subsequent follow-ups, the contractions did not decrease, and he was not conscious at the time when awakened. Common ischemic areas were seen on brain magnetic resonance imaging (MRI). The current status of the patient was considered as spastic tetraplegia. He was followed by tracheostomy during the next two months. On the 110th day of hospitalization, he died due to multiple organ failure secondary to septic shock. Trismus is shown in Figure 1, contraction of the patient is shown in Figure 2.

\section{Case 2}

A 6-year-old refugee male patient was admitted to the pediatric emergency department of another hospital with a history of a punture on his left foot base after stepping on a nail ten days prior to admission. Tetanus vaccine plus $500 \mathrm{IU}$ of TIG were given and then he was included into a vaccination schedule. His wound was superficial and clean, so no antibiotic treatment and surgical wound debridement was required. He was readmitted to the pediatric emergency department with complaints of backpain and difficulty in opening the mouth and swallowing

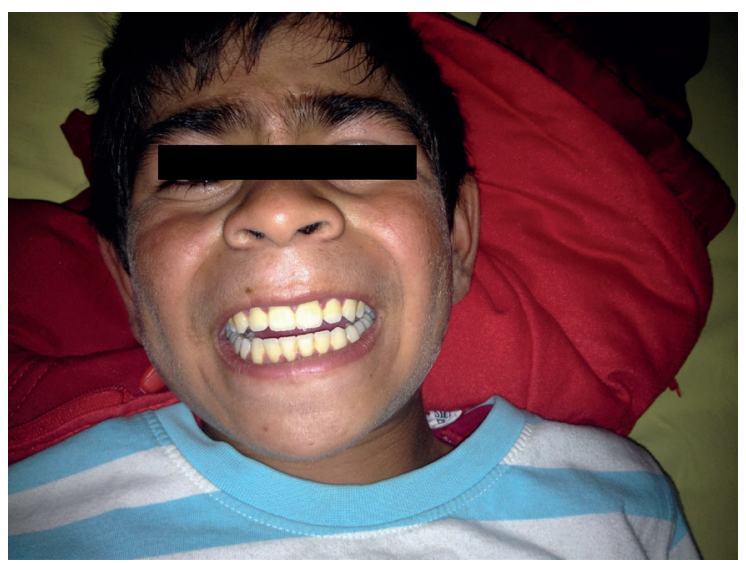

Fig. 1. Trismus of the patient. 


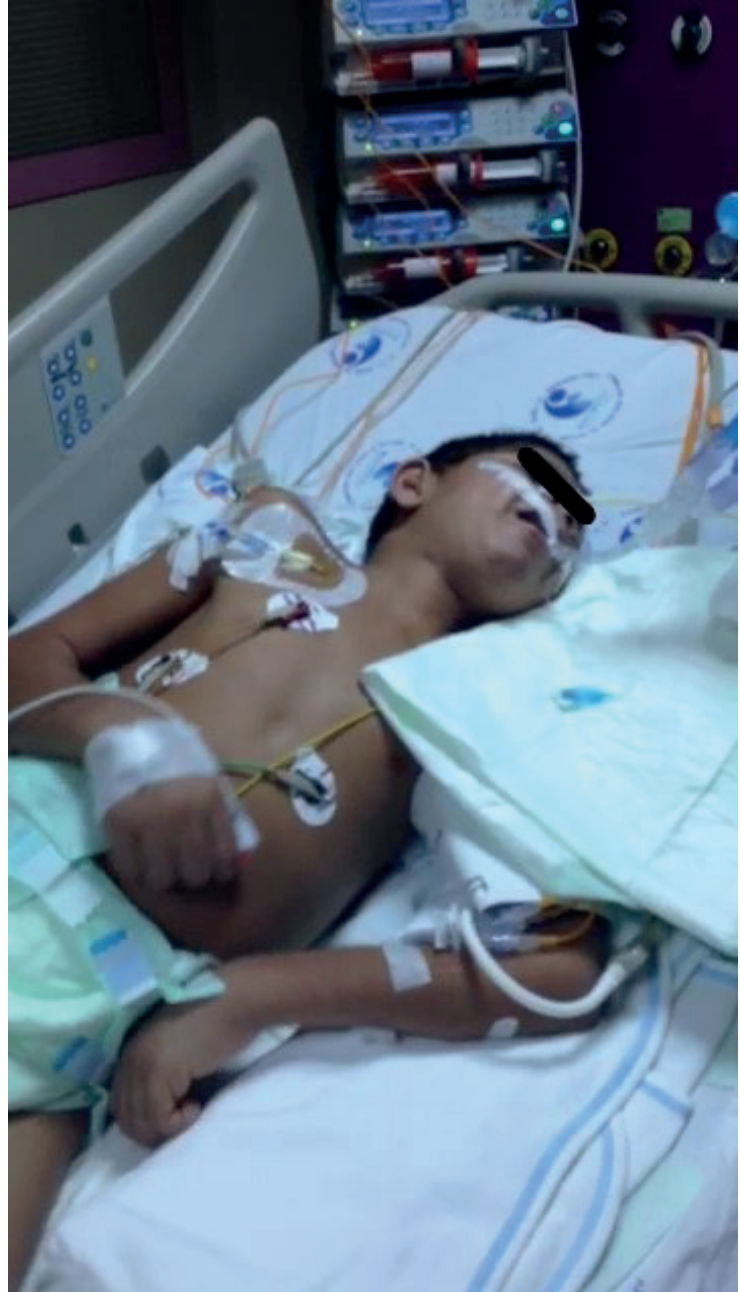

Fig. 2. Contraction of the patient.

for the last few days. At presentation, he was conscious and agitated. His physical examination revealed muscle contractions within all body and lock-jaw. The Glasgow Coma Scale (GCS) score was 13/15. Because of the contraction of the neck and back muscles, he had opisthotonus and respiratory difficulty. Other system examinations and laboratory tests were normal. His medical history indicated that he had not been vaccinated against tetanus. He was hospitalized in the PICU with a preliminary diagnosis of tetanus. Intravenous ampicillin-sulbactam and metronidazole were started and 2500 IU of TIG was administered intramuscularly to the patient that a half dose was injected around the wound areas. The patient was intubated and mechanical ventilation was started due to difficulty of breathing. After intubation, midazolam was started as a sedative, fentanyl was started as an analgesic agent, and rocuronium and magnesium sulphate were started as a muscle relaxant. Baclofen was used enterally via the optimal dose-escalation strategy. The sedation level was monitored by the bispectral index (BIS). Midazolam and thiopental infusions were dynamically regulated so that BSI value remained between 40 and 60 . Brain regional oxygenation was monitored by near-infrared regional spectroscopy (NIRS) receivers attached to the right and left forehead regions. Respiration and circulatory support were provided, so that NIRS value remained over $60 \%$. Brain MRI of the patient was assessed as normal. Despite deep sedation, rocuronium, magnesium sulphate and baclofen treatments, 500 IU of TIG was administered intrathecally to the patient due to resistant contractions observed during daily sedation vacation. Periodic hypertension and autonomic dysfunction were observed. The patient's blood pressure was controlled using amlodipine with esmolol and dexmedetomidine infusions. Sedative and muscle relaxant drugs were discontinued weekly. During the third week of hospitalization, the contractions of the patient were considerably reduced. Therefore, while rocuronium was stopped, sedative and analgesic agents were reduced. On the 26th day of hospitalization, the contractions were rather diminished and the muscle strength was sufficient for spontaneous breathing, so the patient was extubated. Non-invasive mechanical ventilation was primarily used and then oxygen was administered by the mask. On the 32nd day of hospitalization, he was conscious and aware of his surroundings and spontaneously breathed at room air and was well-fed orally. The patient was discharged when the contractions had completely disappeared.

Demographics and treatment approaches used in our patients are shown in detail in Table I.

Written consent was obtained from the parents of the patients participating in this study. 
Table I. Demographics and treatment approaches used both patients.

\begin{tabular}{|c|c|c|}
\hline & Case 1 & Case 2 \\
\hline \multicolumn{3}{|l|}{ Demographics } \\
\hline Age (years) & 12 & 6 \\
\hline $\operatorname{Sex}(M / F)$ & $\mathrm{M}$ & M \\
\hline \multicolumn{3}{|l|}{ Sedatives } \\
\hline Dexmedetomidine & $\mathrm{BI}+\mathrm{AI}$ & $\mathrm{BI}+\mathrm{AI}$ \\
\hline Diazepam & BI & - \\
\hline Sodium thiopental & AI & AI \\
\hline Midazolam & AI & AI \\
\hline \multicolumn{3}{|l|}{ Analgesic Treatment } \\
\hline Fentanyl & $\mathrm{BI}+\mathrm{AI}$ & AI \\
\hline \multicolumn{3}{|l|}{ Muscle Relaxants } \\
\hline Rocuronium & $\mathrm{AI}$ & $\mathrm{AI}$ \\
\hline Magnesium sulphate & Bolus & Infusion \\
\hline \multicolumn{3}{|l|}{ Other Treatments } \\
\hline Baclofen(enteral) & + & + \\
\hline Antibiotics & $\begin{array}{c}\text { Metronidasole, Vancomycin, } \\
\text { Ceftazidime }\end{array}$ & Ampisilin-Sulbactam, Metronidasole \\
\hline TIG(IU) & 6000 & 3500 \\
\hline Mechanical Ventilation(days) & 105 & 26 \\
\hline
\end{tabular}

AI: after intubation, BI: before intubation, TIG: tetanos immunglobulin.

\section{Discussion}

Tetanus is one of the oldest known human diseases. Although there is a great accumulation of information about such an important disease, high mortality rates are not surprising even with the best treatment approaches. Preventative medical approaches for tetanus are far more important than therapeutic approaches. ${ }^{4}$ The best example for this situation could be that both cases were not vaccinated in childhood.

Tetanus is always diagnosed with the history and accompanying clinical findings. ${ }^{3}$ Both cases had a history of "puncture to the foot base by a dirty nail". This condition is usually associated with tetanus and requires urgent hospitalization. However, in our first case, there was no history of hospital admission and tetanus vaccination following the event. Our second case was vaccinated after the event, but the disease still developed. This suggests that childhood vaccinations are more important than post-event vaccinations and TIG application in suspected contaminated injuries, especially in unvaccinated cases, must be made at an early stage.

Although tetanus is an infectious disease, antibiotic treatment takes part at the end in the guidelines and recommended first-line antibiotic is metronidazole. ${ }^{5}$ TIG is one of the most important drugs in the treatment of tetanus, and the recommended dose is 3000-6000 IU given intramuscularly. ${ }^{6}$ In the first case, a total of 6000 IU of TIG was given with two different application methods. Although a total of $3000 \mathrm{IU}$ of TIG was administered in the second case, and 500 IU of the total amount intrathecally, there was no significant difference in clinical course in terms of tetanus. Intrathecal administration of TIG has been a well-known method for many years, but it is still controversial and has not been definitely accepted. ${ }^{7}$ Considering that the second case underwent intrathecal TIG, developed subsequent clinical findings and had an expected recovery time of 4-6 weeks, it is difficult to argue that intrathecal administration 
of TIG led to a significant improvement in clinical course and the recovery period.

Sedative and muscle relaxant drugs are the essential parts of tetanus treatment. Diazepam is frequently used in adults, but its' use in children is limited because it cannot be given by continuous infusion and preservatives have possible toxic effects. ${ }^{8}$ The first case was attempted to be followed with intensive sedation treatment prior to intubation. However, the need for intubation within days had led to the decision of earlier intubation in the management of the second case. Intubation of the patients allows using muscle relaxants and the formation of deeper sedation which leads to the comfort and protection of the patient from damage caused by contractions. Despite the positive clinical benefits, mechanical ventilation can contribute to death and permanent damage due to its' possible complications. In the first case, the development of cardiac arrest may be due to the involvement of the autonomic nervous system in tetanus ${ }^{2}$ and may also be considered as a complication of mechanical ventilation. This can be regarded as the most important "event" for the negative outcome of the case. The involvement of the autonomic nervous system is condition reported in tetanus cases ${ }^{2}$, and hypertension and tachycardia were seen in both cases. Dexmedetomidine is a drug which has sedative, analgesic and sympatholytic effects since it is a centrally acting alpha- 2 agonist. ${ }^{9}$ In the second case, we observed esmolol infusion showed positive effects on the attacks of hypertension and tachycardia.

Magnesium used to prevent contraction and stiffness in the treatment of tetanus prevents the effect of calcium at the neuromuscular junction. There are publications in the literature showing that magnesium is used in tetanus contractions, which do not respond to anticonvulsant and muscle relaxant drugs and as a result contractions were significantly suppressed. ${ }^{10,11}$ We used magnesium sulphate to control refractory contractions in both cases.
The development of monitoring techniques in intensive care units seems to increase the chances of successful patient management. ${ }^{12}$ We think that the BIS and NIRS monitors, which created differences in the management of these two cases are three years apart, may have had a critical effect on the positive outcome of the second case. The BIS monitoring, increases the patient's comfort, but also prevents the administration of excessive sedation. Similarly, the NIRS monitor gives information on cerebral blood flow and oxygen delivery and thus enables appropriate hemodynamic and ventilatory support and makes it possible to follow their results. ${ }^{12}$ For these reasons, we believe that advanced monitoring techniques in tetanus cases may improve treatment success compared with standard approaches

Consequently, it should be kept in mind that tetanus with high mortality and morbidity rates can be seen in countries where immigration takes place. Although it has been presented as a forgotten disease in the title, it should be known that the disease will be seen more frequently if children are not vaccinated. Also injury cases should be carefully questioned in terms of the need for tetanus vaccination and TIG, and they should be implemented without delay. Identification of unvaccinated people and administration of vaccination programs could reduce the possibility of seeing a new case. We think that the use of early intubation and advanced monitoring techniques can provide positive contributions to reducing death and illness in addition to vaccination, TIG, sedation and muscle relaxant treatments.

\section{REFERENCES}

1. Stock I. Tetanus and clostridium tetani-a brief review. Med Monatsschr Pharm 2015; 38: 57-60.

2. Yalcın I, Salman N, Somer A. Bakteri enfeksiyonları. İçinde: Neyzi O, Ertuğrul T (eds). Pediatri Cilt 1-2 (4. Baskı), Ankara: Nobel Tip Kitabevi, 2010: 613-616.

3. Aydın TT, Bayhan GI, Afsarlar CE, Oz FN, Akansel AR, Tanir G. A report of two pediatric tetanus cases. Turk J Pediatr 2011; 53: 437-440. 
4. Tetanus vaccines: WHO position paper-February 2017. Wkly Epidemiol Rec 2017; 92: 53-76.

5. RodrigoC,FernandoD, RajapakseS. Pharmacological management of tetanus: an evidence based review. Crit Care 2014; 18: 217.

6. American Academy of Pediatrics. Tetanus (Lockjaw). In: Pickering LK, Baker CJ, Kimberlin DW, Long SS (eds). Red Book: 2012 Report of the Committee on Infectious Diseases (29th ed). Elk GroveVillage, IL: American Academy of Pediatrics, 2012: 707-712.

7. Kabura L, Ilibagiza D, Menten J, Van den Ende J. Intrathecal vs. intramuscular administration of human antitetanus immunoglobulin or equine tetanus antitoxin in the treatment of tetanus: a metaanalysis. Trop Med Int Health 2006; 11: 1075-1081.

8. McTague A, Martland T, Appleton R. Drug management for acute tonic-clonic convulsions including convulsive status epilepticus in children. Cochrane Database Syst Rew 2008; CD001905.
9. Burns J, Jackson K, Sheehy KA, Finkel JC, Quezado $Z M$. The use of dexmedetomidine in pediatric palliative care: a preliminary study. J Palliat Med 2017; 20: 779-783.

10. Katı I, Goktas U, Cagan E, Guzel A, Bartın S. The effect of magnesium sulfate on uncontrollable contractions in a tetanus case. Pediat Emerg Care 2012; 28: 366-367.

11. Attygalle D, Rodrigo N. Magnesium as first line therapy in the management of tetanus: a prospective study of 40 patients. Anaesthesia 2002; 57: 811-817.

12. Hayashida M, Kin N, Tomioka T, et al. Cerebral ischaemia during cardiac surgery in children detected by combined monitoring of BIS and nearinfrared spectroscopy. Br J Anaesth 2004; 92: 662-669. 